\title{
Risk Factors for Shoulder Pain in Japanese Badminton Players: A Quantitative-Research Survey
}

\author{
Yuki Warashina ${ }^{1,3}$, Ryo Ogaki ${ }^{2}$, Akemi Sawai ${ }^{1}$, Hitoshi Shiraki ${ }^{1,3}$ and Shumpei Miyakawa ${ }^{1,3}$ \\ 1. Department of Sports Medicine, Graduate School of Comprehensive Human Sciences, University of Tsukuba, Ibaraki 305-8577, \\ Japan \\ 2. Sports Research and Development Core, University of Tsukuba, Ibaraki 305-8574, Japan \\ 3. Faculty of Health and Sport Sciences, University of Tsukuba, Ibaraki 305-8574, Japan
}

\begin{abstract}
This study aimed to elucidate the key risk factors for shoulder pain in Japanese middle school-, high school-, and university-level badminton players and use a regression formula to predict the probability of shoulder pain onset based on risk factor combinations. Based on a self-report questionnaire survey, we included 461 individuals (102 middle school students, 134 high school students, and 225 university students) with a history of shoulder pain in the previous year and 541 individuals with no shoulder pain in the previous year. Then, we performed logistic regression analysis of individual characteristics and history of shoulder pain to analyze the factors associated with shoulder pain onset. Analysis of patients excluding those currently experiencing shoulder pain indicated that a history of shoulder pain (> 1 year previously) predicted shoulder pain onset. Analysis of patients excluding those with a history of shoulder pain indicated that undergoing care, being of university age, having a long history of competing, and short cool-down times were associated with recurrent pain. However, results were different for each age group. The fact that half the Japanese middle school, high school, and university badminton players had a history of shoulder pain suggests that they will experience recurrent shoulder pain. The results also suggest that different factors are important in each age group. Thus, it is critical to establish evidence-based countermeasures to prevent shoulder pain that interferes with play.
\end{abstract}

Key words: Shoulder pain, badminton, questionnaire, epidemiology, logistic regression analysis.

\section{Introduction}

Badminton is a popular sport that is enjoyed by both sexes and people of all ages around the world [1]. Unlike recreational badminton, competitive badminton places a great deal of load on the player due to vigorous upper arm, trunk, and leg movements [1]. Consequent to the vigorous movements and exercise load, many players suffer trauma and injury. A survey of Japanese university-level badminton players indicated that $50 \%$ suffered injuries in high school compared with $25 \%$ in middle school and/or university [2]. Based on these data, we determined that an age-based survey of the risk of injury was required.

Hitting the shuttlecock in badminton places a high

Corresponding author: Shumpei Miyakawa, M.D., Ph.D., professor, research field: sport medicine. degree of force on the shoulder joint [3], and a characteristic feature of this sport is that it requires more shoulder mobility than other sports. Arm rotation, particularly at the shoulder, is an important component of strokes, and stroke repetition increases the load on the shoulder. In a survey of 469 elite badminton players in Malaysia [4], 18.1\% had arm injuries, of which $36.9 \%$ were shoulder injuries. Similarly, a survey of 57 Japanese Olympic athletes found that $18 \%$ had shoulder injuries [5]. Badminton strokes require the shoulder joint to transmit energy from the legs and trunk to the arms $[6,7]$, and poor coordination of the kinetic chain due to shoulder pain can negatively affect performance. Unfortunately, badminton-related arm injuries have been reported to persist for prolonged periods [8].

There are many reports of trauma and injury 
associated with badminton [9-15], but only few of these have reported pain not associated with serious injury. In a survey of shoulder pain among 188 competitors at international meets [16], 97 players (52\%) reportedly had current pain, and more than half of these reported that the pain affected their daily lives and ability to play. In a study by our group in 2015, we elucidated that approximately half (53.3\%) of the 1,002 badminton players we questioned in Japan (ranging from middle school students to university students) had a history of shoulder pain [17]. This research also indicated that there were differences between those competing at middle school (58.4\%), high school (38.2\%), and university (66.6\%) levels. Approximately half $(48.4 \%)$ of the 461 players who reported to have pain within the previous year also reported to have problems with their ability to play, with a similar prevalence to that previously reported among the competitors at international meets [16]. We believe that preventing shoulder pain at junior and youth levels (i.e., middle school, high school, university levels), to which players who attend international meets belong, could be an important target in reducing the burden of this common sports-related problem.

Shoulder pain associated with competitive badminton not only has a negative effect on players' performances but also has a major negative effect on their daily lives. Preventing pain, particularly among badminton competitors at junior and youth levels, could improve performance and enjoyment throughout life. However, the causes of shoulder pain remain unknown in this group, which precludes the development of evidence-based preventive measures among middle school-, high school-, and university-level badminton players, who make up nearly $70 \%$ of all competitive badminton players in Japan. We believe that identifying important risk factors for injury based on age and sex can ameliorate this situation. Indeed, to establish effective preventive measures, it is necessary to understand the correlation between factors associated with the onset of shoulder pain based on the analysis of personal data and the types of pain experienced by large numbers of players. It is also necessary to quantify the likelihood of pain onset.

In this study, we aimed to elucidate the relative importance of individual risk factors associated with shoulder pain experienced by Japanese competitive middle school-, high school-, and university-level badminton players, and to predict the likelihood of pain onset based on combinations of these factors using regression analysis.

\section{Materials and Methods}

\subsection{Study Design \& Subjects}

The survey period was between October and December 2011. Subjects were Japanese middle school, high school, and university students from competitive badminton teams. Requests to participate in our survey were sent to 15 middle schools, 23 high schools, and 16 universities in the Kanto region of Japan. The original, self-administered survey was distributed to 1,410 individuals, of whom 1,009 responded. Effective responses were received from 1,002 individuals (71\%; 202 middle school students, 411 high school students, and 389 university students).

Before completing the survey, respondents were provided with both oral and written explanations of the study, and written consent was obtained from their representatives. The questionnaire also indicated that survey participation was voluntary and that responses would be made anonymous to protect the privacy of respondents. The study was conducted with the approval of the Institutional Review Board of the Graduate School of Comprehensive Human Sciences, University of Tsukuba.

\subsection{Procedures}

Representatives of the potential badminton teams at different educational institutions (i.e., middle schools, high schools, and universities) were contacted by phone or by opportunistic sampling (referral by 
acquaintance) to identify institutions that would participate in the study. The selection criteria for schools were as follows: badminton practice once per week on average, team practice for $1 \mathrm{~h}$ continuously per practice session on average, and teams with at least five players registered with the Nippon Badminton Association. Educational institutions that met these criteria and provided consent were included. Depending upon the circumstances of each team, we used the drop-off method (1 middle school, 2 universities), group survey method (6 middle schools, 2 high schools, 6 universities), or mail-in method (8 middle schools, 21 high schools, 8 universities) to distribute and recover questionnaires.

\subsection{Questionnaire}

The questionnaire survey included items related to the individuals themselves and to whether they had shoulder pain (within the previous year) as follows:

\subsubsection{Personal Details}

Basic data such as sex, age, height, weight, dominant hand, competition history ( $<3$ years, 3-6 years, $\geq 6$ years), and competition level (e.g., if they had ever played in national games) were collected. Also, specific data related to competition were collected, including racket string tension (in pounds), style of play, strongest event(s), amount of practice (mean number of times per week, mean number of hours per day, number of hits, number of misses), warm-up and cool-down times (overall and for each arm), frequency of shoulder care, and whether shoulder joint pain had been experienced $\geq 1$ year in the past on the dominant side (the racket-hand side).

\subsubsection{Shoulder Pain}

We asked whether the shoulder joint pain was present on the dominant side, defined as shoulder joint pain on the racket-hand side within the previous year and shoulder joint pain more than 1 year previously.

\subsection{Statistical Analyses}

Extraction of factors related to shoulder joint pain was performed based on age group according to the following six-step procedure. First, comparison of personal data for the shoulder pain and no shoulder pain groups based on age was done by univariate analysis of each item, with items removed if they showed no clear correlation. Second, logistic regression analysis was performed with the personal data items as the independent variables and the presence or absence of shoulder joint pain within the previous year as the dependent variable. We used the variable increase and decrease method, with the likelihood ratio as our standard. Variable selection was performed using the Wald test. Third, assessment of the model-goodness-of-fit of the regression formula was performed using the omnibus test of model coefficients, model contribution, and the Hosmer-Lemeshow goodness-of-fit test, with statistical significance set at < $5 \%$. Fourth, we created a contingency table with the cut-off level for predictive probability set at 0.50 and then calculated the sensitivity, specificity, positive predictive value, negative predictive value, and predictive accuracy of the regression formula. Fifth, we used the Wald test to determine whether the onset of each related factor had a significant influence at the 5\% level. Finally, odds ratios (ORs) and 95\% confidence intervals (CIs) were used to indicate the influence of factors related to pain onset. All analyses were performed using SPSS version 22.0 (SPSS Inc, Chicago, IL, USA).

\section{Results and Analysis}

The survey results show that 461 players (102 middle school students, 134 high school students, and 225 university students) had experienced shoulder joint pain within the previous year. The results of logistic regression analysis for the entire survey population and based on the three age groups are described below.

The only factor related to pain onset both overall and based on the age subgroup was a history of shoulder joint pain $\geq 1$ year in the past ("shoulder pain history") after excluding those currently experiencing pain. The 
"shoulder pain history" factor was excluded as an independent variable in subsequent analyses. Table 1 shows factors that were significantly related to the onset of shoulder joint pain in the overall population.

We rejected the hypothesis that the logistic regression formula would not be useful in predicting ( $p$ $<0.05$; Table 2) and showed its contribution to be between 0.068 and 0.091 (Table 3 ). However, we were not able to confirm goodness-of-fit ( $p>0.05$; Table 4). When we created a contingency table with a cut-off value for regression formula P1 set at 0.5 (Table 5), we obtained the predictive values shown in Table 6 .

\subsection{Middle School Students}

Table 7 shows that the factors significantly related to the onset of shoulder joint pain in middle school students were, in order of higher to lower ORs, "core muscle training," "shoulder stretches," "competition history," and "C-down time." When we created the contingency table with the cut-off value for regression

Table 1 Results of logistic regression analysis: regression formula 1.

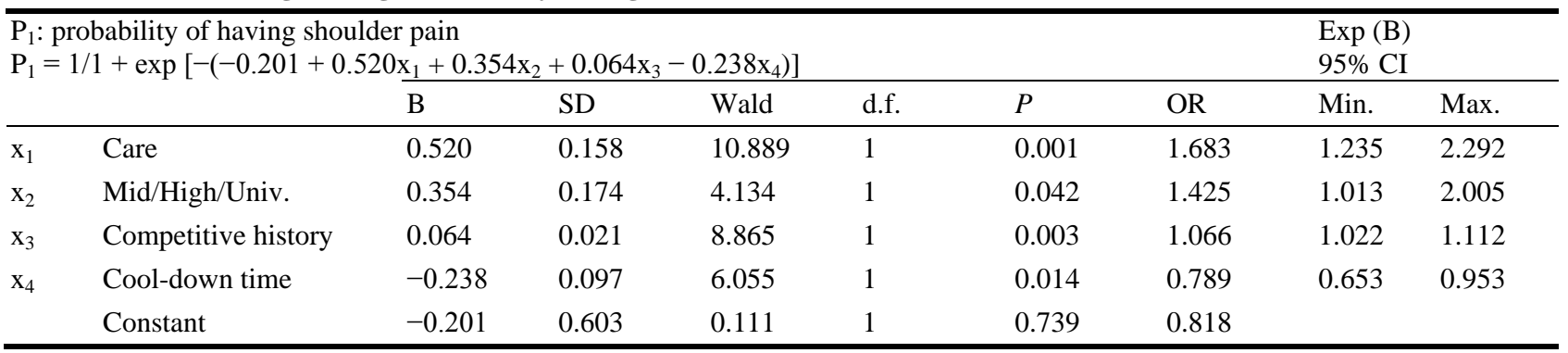

$\mathrm{B}=$ coefficient $\mathrm{SD}=$ standard deviation; d.f. = degree of freedom; $\mathrm{OR}=$ Odds ratio; $\mathrm{CI}=$ confidence interval.

Table 2 Omnibus test of the model coefficient in regression formula 1.

\begin{tabular}{lll}
\hline$-2 \log$ likelihood & Cox-Snell R-squared & Nagelkerke R-squared \\
\hline $1311.659^{\mathrm{a}}$ & 0.068 & 0.091 \\
\hline
\end{tabular}

Table 3 Contribution of regression formula 1.

\begin{tabular}{lll}
\hline Chi-square & Degree of freedom & $P$-value \\
\hline 7.675 & 8 & 0.466 \\
\hline
\end{tabular}

Table 4 Hosmer-Lemeshow test of regression formula 1.

\begin{tabular}{lll}
\hline & Predicted negative & Predicted positive \\
\hline Observed negative & 392 & 149 \\
Observed positive & 232 & 229 \\
\hline
\end{tabular}

Table 5 Validity of regression formula 1.

\begin{tabular}{llll}
\hline & Chi-square & Degree of freedom & $P$-value \\
\hline Step & 71.014 & 4 & 0.000 \\
Block & 71.014 & 4 & 0.000 \\
Model & 71.014 & 4 & 0.000 \\
\hline
\end{tabular}

Table 6 Predictive value of regression formula 1.

\begin{tabular}{ll}
\hline Sensitivity & $49.7 \%$ \\
\hline Specificity & $72.5 \%$ \\
Positive predictive value & $60.6 \%$ \\
Negative predictive value & $62.8 \%$ \\
Accuracy & $62.0 \%$ \\
\hline
\end{tabular}


formula P1 set at 0.5 , formula 2 showed that onset prediction sensitivity was $66.7 \%$, specificity was $72.0 \%$, positive predictive value was $70.8 \%$, negative predictive value was $67.9 \%$, and accuracy was $69.3 \%$.

We rejected the hypothesis that the logistic regression formula would not be useful in predicting ( $p$ $<0.05$; Table 8) and showed its contribution to be between 0.165 and 0.220 (Table 9). However, we were not able to confirm goodness-of-fit $(p>0.05$; Table 10). When we created a contingency table with a cut-off value for regression formula $\mathrm{P} 2$ set at 0.5
(Table 11), we obtained the predictive values shown in Table 12 .

\subsection{High School Students}

The factors significantly correlated with the onset of shoulder joint pain in high school students are summarized in Table 13.

We rejected the hypothesis that the logistic regression formula would not be useful in predicting ( $p<0.05$; Table 14) and showed its contribution to be between 0.081 and 0.113 (Table 15). However, we

Table 7 Results of logistic regression analysis: regression formula 2.

\begin{tabular}{|c|c|c|c|c|c|c|c|c|c|}
\hline \multicolumn{7}{|c|}{$\begin{array}{l}\mathrm{P}_{2}: \text { probability of having shoulder pain } \\
\mathrm{P}_{2}=1 / 1+\exp \left[-\left(2.591+1.957 \mathrm{x}_{1}+0.933 \mathrm{x}_{2}-0.471 \mathrm{x}_{3}-0.155 \mathrm{x}_{4}\right)\right]\end{array}$} & \multicolumn{3}{|c|}{$\begin{array}{l}\operatorname{Exp}(\mathrm{B}) \\
95 \% \mathrm{CI} \\
\end{array}$} \\
\hline & & $\mathrm{B}$ & SD & Wald & d.f. & $P$ & OR & Min. & Max. \\
\hline $\mathrm{x}_{1}$ & Core Muscle Tr. & 1.957 & 0.608 & 10.368 & 1 & 0.001 & 7.077 & 2.151 & 23.289 \\
\hline $\mathrm{x}_{2}$ & Shoulder stretch & 0.933 & 0.318 & 8.621 & 1 & 0.003 & 2.543 & 1.364 & 4.740 \\
\hline $\mathrm{x}_{3}$ & Cool-down time & -0.471 & 0.232 & 4.134 & 1 & 0.042 & 0.624 & 0.396 & 0.983 \\
\hline \multirow[t]{2}{*}{$\mathrm{x}_{4}$} & Competitive history & -0.155 & 0.069 & 4.986 & 1 & 0.026 & 0.856 & 0.747 & 0.981 \\
\hline & Constant & 2.591 & 1.414 & 3.357 & 1 & 0.067 & 13.339 & & \\
\hline
\end{tabular}

$\mathrm{B}=$ coefficient; $\mathrm{SD}=$ standard deviation; d.f. = degree of freedom; $\mathrm{OR}=$ Odds ratio; $\mathrm{CI}$ = confidence interval.

Table 8 Omnibus test of the model coefficient in formula 1.

\begin{tabular}{llll}
\hline & Chi-square & Degree of freedom & $P$-value \\
\hline Step & 36.370 & 4 & 0.000 \\
Block & 36.370 & 4 & 0.000 \\
Model & 36.370 & 4 & 0.000 \\
\hline
\end{tabular}

Table 9 Contribution of formula 2.

\begin{tabular}{lll}
\hline$-2 \log$ likelihood & Cox-Snell R-squared & Nagelkerke R-squared \\
\hline $243.642^{\mathrm{a}}$ & 0.165 & 0.220 \\
\hline
\end{tabular}

Table 10 Hosmer-Lemeshow test of formula 2.

\begin{tabular}{lll}
\hline Chi-square & Degree of freedom & $P$-value \\
\hline 7.857 & 8 & 0.448 \\
\hline Table $\mathbf{1 1} \quad$ Validity of regression formula $\mathbf{2 .}$ & \\
\hline & Predicted negative & Predicted positive \\
\hline Observed negative & 72 & 28 \\
Observed positive & 34 & 68 \\
\hline
\end{tabular}

Table 12 Predictive value of regression formula 2.

\begin{tabular}{ll}
\hline Sensitivity & $66.7 \%$ \\
\hline Specificity & $72.0 \%$ \\
Positive predictive value & $70.8 \%$ \\
Negative predictive value & $67.9 \%$ \\
Accuracy & $69.3 \%$ \\
\hline
\end{tabular}


were not able to confirm goodness-of-fit $(p>0.05$; Table 16). When we created a contingency table with a cut-off value for regression formula P3 set at 0.5 (Table 17), we obtained the predictive values shown in Table 18.

\subsection{University Students}

The factors significantly correlated to the onset of shoulder joint pain in university students are shown in
Table 19.

We rejected the hypothesis that the logistic regression formula would not be useful in predicting ( $p$ $<0.05$; Table 20) and showed its contribution to be between 0.076 and 0.103 (Table 21). However, we were not able to confirm goodness-of-fit ( $p>0.05$; Table 22). When we created a contingency table with a cut-off value for regression formula $\mathrm{P} 4$ set at 0.5 (Table 23), we obtained the predictive values shown in Table 24.

Table 13 Results of logistic regression analysis: regression formula 3.

\begin{tabular}{|c|c|c|c|c|c|c|c|c|c|}
\hline \multicolumn{7}{|c|}{$\begin{array}{l}\mathrm{P}_{3} \text { : probability of having shoulder pain } \\
\mathrm{P}_{3}=1 / 1+\exp \left[-\left(-4.051+0.814 \mathrm{x}_{1}+0.690 \mathrm{x}_{2}+0.681 \mathrm{x}_{3}+0.243 \mathrm{x}_{4}\right)\right]\end{array}$} & \multicolumn{3}{|c|}{$\begin{array}{l}\operatorname{Exp}(\mathrm{B}) \\
95 \% \mathrm{CI}\end{array}$} \\
\hline & & $\mathrm{B}$ & $\mathrm{SD}$ & Wald & d.f. & $P$ & OR & Min & Max. \\
\hline $\mathrm{x}_{1}$ & National meets & 0.814 & 0.390 & 4.359 & 1 & 0.037 & 2.257 & 1.051 & 4.848 \\
\hline$x_{2}$ & Massage & 0.690 & 0.228 & 9.142 & 1 & 0.002 & 1.994 & 1.275 & 3.118 \\
\hline$x_{3}$ & $\begin{array}{l}\text { Mean hours } \\
\text { Practice/day }\end{array}$ & 0.681 & 0.196 & 12.027 & 1 & 0.001 & 1.976 & 1.345 & 2.903 \\
\hline \multirow[t]{2}{*}{$\mathrm{x}_{4}$} & Warm-up time & 0.243 & 0.082 & 8.668 & 1 & 0.003 & 1.275 & 1.084 & 1.498 \\
\hline & Constant & -4.051 & 0.721 & 31.548 & 1 & 0.000 & 0.017 & & \\
\hline
\end{tabular}

$\mathrm{B}=$ coefficient $\mathrm{SD}=$ standard deviation; d.f. = degree of freedom; $\mathrm{OR}=$ Odds ratio $\mathrm{CI}=$ confidence interval.

Table 14 Omnibus test of the model coefficient for formula 3.

\begin{tabular}{llll}
\hline & Chi-square & Degree of freedom & $P$-value \\
\hline Step & 34.756 & 4 & 0.000 \\
Block & 34.756 & 4 & 0.000 \\
Model & 34.756 & 4 & 0.000 \\
\hline
\end{tabular}

Table 15 Contribution of formula 3.

\begin{tabular}{lll}
\hline$-2 \log$ likelihood & Cox-Snell R-squared & Nagelkerke R-squared \\
\hline $484.201^{\mathrm{a}}$ & 0.081 & 0.113 \\
\hline
\end{tabular}

Table 16 Hosmer-Lemeshow test of formula 3.

\begin{tabular}{lll}
\hline Chi-squared & Degree of freedom & $P$-value \\
\hline 5.966 & 8 & 0.651 \\
\hline
\end{tabular}

Table 17 Validity of regression formula 3.

\begin{tabular}{lll}
\hline & Predicted negative & Predicted negative \\
\hline Observed negative & 258 & 19 \\
Observed positive & 99 & 35 \\
\hline
\end{tabular}

Table 18 Predictive value of regression formula 3

\begin{tabular}{ll}
\hline Sensitivity & $26.1 \%$ \\
\hline Specificity & $93.1 \%$ \\
Positive predictive value & $64.8 \%$ \\
Negative predictive value & $72.3 \%$ \\
Accuracy & $71.3 \%$ \\
\hline
\end{tabular}


Table 19 Results of logistic regression analysis: regression formula 4.

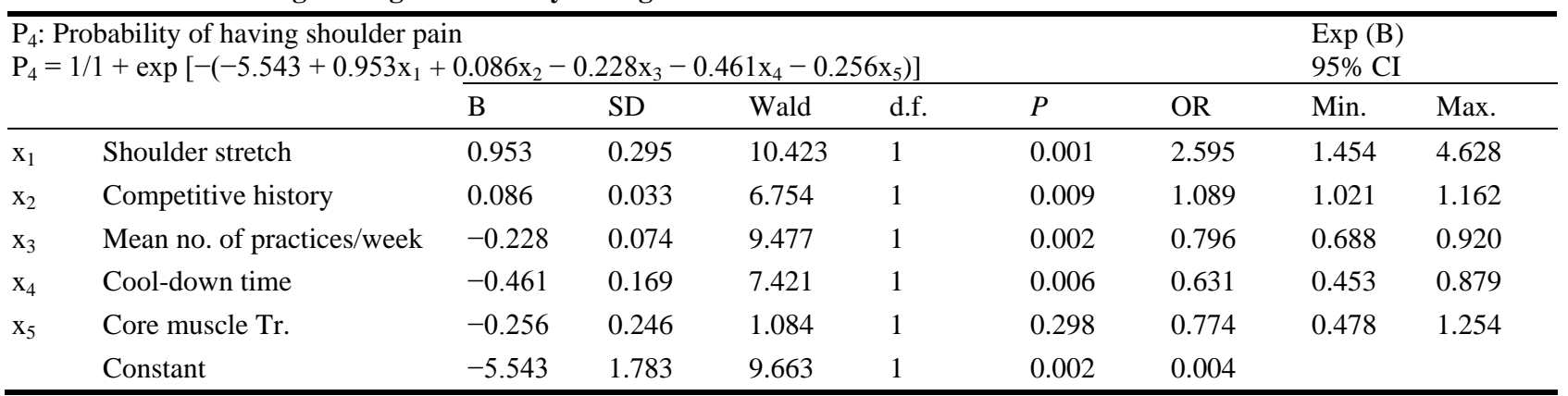

$\mathrm{B}=$ coefficient; $\mathrm{SD}=$ standard deviation; d.f. = degree of freedom; $\mathrm{OR}=$ Odds ratio; $\mathrm{CI}=$ confidence interval.

Table 20 Omnibus test for the model coefficient of formula 4.

\begin{tabular}{llll}
\hline & Chi-squared & Degree of freedom & $P$-value \\
\hline Step & 30.928 & 5 & 0.000 \\
Block & 30.928 & 5 & 0.000 \\
Model & 30.928 & 5 & 0.000 \\
\hline
\end{tabular}

Table 21 Contribution of formula 4.

\begin{tabular}{lll}
\hline$-2 \log$ likelihood & Cox-Snell R-squared & Nagelkerke R-squared \\
\hline $498.735^{\mathrm{a}}$ & 0.076 & 0.103 \\
\hline
\end{tabular}

Table 22 Hosmer-Lemeshow test of formula 4.

\begin{tabular}{lll}
\hline Chi-squared & Degree of freedom & $P$-value \\
\hline 9.814 & 8 & 0.278 \\
\hline
\end{tabular}

Table 23 Validity of regression formula 4.

\begin{tabular}{lll}
\hline & Predicted negative & Predicted negative \\
\hline Observed negative & 62 & 102 \\
Observed positive & 38 & 187 \\
\hline
\end{tabular}

Table 24 Predictive value of regression formula 4.

\begin{tabular}{ll}
\hline Sensitivity & $83.1 \%$ \\
\hline Specificity & $37.8 \%$ \\
Positive predictive value & $64.7 \%$ \\
Negative predictive value & $62.0 \%$ \\
Accuracy & $64.0 \%$ \\
\hline
\end{tabular}

\section{Discussion}

We used logistic regression analysis to assess the associations between shoulder joint pain and personal characteristics, aiming to identify factors that predicted the onset of shoulder joint pain. This method was originally used in cohort studies, but in recent years, it has often been used in other epidemiological studies [18]. We believe that logistic regression analysis should be more actively used to analyze data in sports medicine because it allows the investigation of multiple items that describe target variables. Logistic regression analysis also provides a numerical outcome (the OR) for the degree of influence independent variables have on a binary dependent variable. Given that the method does not place strict restrictions on the measurement and distribution of independent variables, such regression analyses are highly versatile when 
applied to environmental data with high variability. In this study, we identified a history of pain as the only factor related to future pain onset and excluded it as an independent variable. This result was consistent with that of a previous report, which showed that the factor with the highest correlation to the onset of injuries related to ball games was a history of pain of $\geq 1$ year in the past [18]. This supports the idea that once pain has developed, there is a high probability that it will occur repeatedly in the future.

Care, cool-down time, age group, and competitive history were related to recurrent pain overall. Competitors who received care had a 1.683 times higher odds of developing pain than those who did not receive care, whereas those who had shorter post-training cool-down times had a lower likelihood of developing pain than those who had longer post-training cool-down times $(\mathrm{OR}=0.789)$. These results probably reflect the fact that those who experienced pain were more likely to perform care activities and engage in longer cool-down times. Based on age group, university students were 1.425 times more likely to develop pain than middle and high school students. Also, as competitive history increased each year, the likelihood of developing shoulder joint pain increased by 1.066 . These results suggest that the load on the shoulder joint accumulates as players' age and that consequently, the number of players who experience pain increases.

In middle school students, the factors related to recurrent pain were core muscle training, shoulder stretching, cool-down time, and competitive history. Players who engaged in core muscle training were markedly more likely to develop shoulder pain than those who did not $(\mathrm{OR}=7.077)$. Similarly, those who engaged in shoulder stretching were 2.543 times more likely to develop shoulder pain than those who did not, whereas those who had shorter post-training cool-down times were less likely to develop shoulder pain than those who had longer post-training cool-down time $(\mathrm{OR}=0.624)$. As with the overall results, we surmise that those who experienced pain engaged in conditioning activities designed to alleviate the pain and that this inevitably increased cool-down times. With regards to the core muscle training result, we believe that many players were already suffering from pain before they started training for the first time and that preventive training had not yet become established. With each additional year of participation in competitive badminton, we showed that the likelihood of developing shoulder pain was associated with an OR of 0.856 . Competitive history was also related to pain onset for the entire population, but because the odds ratio was $<1$, it was only correlated with the presence of slight pain. This pain could have been caused by continuous practice soon after players started to engage in competitive badminton, which is therefore before they had become accustomed to the exertion.

In high school students, the factors related to recurrent pain were self-massage, mean daily hours of practice, warm-up time, and participation in national meets. Those who practiced self-massage were 1.994 times more likely to develop shoulder pain than those who did not, and those who had shorter warm-up times were 1.275 times more likely to develop shoulder pain than those who had longer warm-up times. We assume that those who experienced pain engaged in care designed to alleviate the pain and that unlike players in the other age groups, high school students with pain were more likely to neglect warm-up as part of their conditioning. It was also notable that those who practiced more were 1.976 times more likely to develop pain and that those who participated in national meets were 2.257 times more likely to develop shoulder pain. Thus, pain may be caused by overloading the body during daily practice or by physical, technical, or strategic factors required at higher levels of play (i.e., national level).

In university students, the factors related to recurrent pain were shoulder stretching, cool-down time, competitive history, and number of practice sessions per week. Those who engaged in shoulder stretching 
were 2.595 times more likely to develop shoulder pain than those who did not, whereas those who had shorter cool-down times were less likely to develop pain than those who had longer cool-down times $(\mathrm{OR}=0.631)$. These results indicate that those with pain engaged in meticulous care to alleviate pain and used increased cool-down time as a form of conditioning. The OR for competitive history indicated that with each additional year of participation in competitive badminton, players were 1.089 times more likely to develop shoulder pain. This was probably because load on the shoulder joint accumulates as the time engaged in competitive badminton increases. Those who engaged in more practice were less likely to develop pain $(\mathrm{OR}=0.796)$. We assume that this was because of inappropriate levels of daily training intensity or lack of awareness that the amount of preparation, care, conditioning, and rest affect recovery needs when practicing at a higher intensity.

Our results suggest that awareness and implementation rates for care and conditioning before badminton competitions were low among competitors. These rates were especially lower among younger players, with different trends evident in each age group. Overall, it seems that care and conditioning are thought of as measures to be taken after pain develops, with the idea of preemptive care and conditioning not being prevalent at any age, despite evidence that it can be too late to prevent future pain after pain is established. It has been shown that injury numbers decreased when elite Japanese badminton competitors engaged in muscle training, stretching, and other training guided by specialists [19]. The present results support that finding and indicate the need to prioritize core muscle training and stretching as preventive measures.

In theory, one would expect that injury rates increase as the age of the players increase, but we found that it was the simultaneous effect of multiple factors from young ages that affected the development of shoulder pain. However, the retrospective cross-sectional design means that we cannot be certain whether the factors related to pain onset were the result or the cause of that pain. Thus, it will be necessary to conduct prospective longitudinal studies that include pathological findings, shoulder alignment, and other factors.

Currently, several measures are used to manage pain at competitive venues [19]. However, none of these is based on observational evidence derived from studies. The identification of personal characteristics related to pain onset (e.g., sex, age, and height) and of persons at high risk of developing pain can provide valuable data that can be used to establish evidence-based preventive measures. In addition, the identification of non-fixed factors (e.g., weight, play style, and daily use of ice) can help with this goal. The present study therefore gives preliminary data in the study of such factors. This descriptive epidemiological investigation of shoulder pain has ascertained the current situation based on age groups, but it is now necessary to conduct interventional examinations of factors that can be used to predict pain onset and verify the correlation between the predictive factors and pain onset. We also recommend investigating relevant physical and kinetic factors in addition to the items included in this study to provide more specific inferences about the factors related to pain onset. Finally, because this epidemiological study only ascertained the current state of shoulder pain by patient self-report, it is highly likely that the definition of shoulder pain was not consistent. Future studies should therefore investigate shoulder pain based on the severity level.

\section{Conclusions}

Badminton players of all ages often have a history of shoulder pain, suggesting that shoulder pain is a serious problem in this sport. Examining the factors related to pain at each age level indicates that there are significant differences in practice environments, physical characteristics, and implementation of care. Approximately half of all junior and youth badminton players in Japan have a history of shoulder pain, suggesting that players in these age groups will develop 
shoulder pain in the future. However, the causes of shoulder pain appear to differ based on age, implying that it will be necessary to establish preventive measures that target the specific needs of each age group.

\section{Acknowledgments}

No financial assistance or grant support was obtained for this study. The authors appreciate the contribution of all participants.

\section{References}

[1] Sakurai, S., and Ohtsuki, T. 2000. "Muscle Activity and Accuracy of Performance of the Smash Stroke in Badminton with Reference to Skill and Practice." Journal of Sports Sciences 18 (11): 901-14.

[2] Suita, M., and Miyanaga, Y. 2003. "Injury Characteristics among College Badminton Players-Analysis of Questionnaire on the History of Injuries." Ibaraki Journal of Health and Sport Sciences 20: 11-20.

[3] Andersen, L. L., Larsson, B., Overgaard, H., and Aagaard, P. 2007. "Torque-Velocity Characteristics and Contractile Rate of Force Development in Elite Badminton Players." European Journal of Sport Science 7 (3): 127-34.

[4] Shariff, A. H., George, J., and Ramlan, A. A. 2009. "Musculoskeletal Injuries among Malaysian Badminton Players.” Singapore Medical Journal 50 (11): 1095-7.

[5] Ogiuchi, T., Muneta, T., Yagishita, K., and Yamamoto, H. 1998. "Sports Injuries in Elite Badminton Players." Japanese Journal of Orthopaedic Sports Medicine 18: 343-8.

[6] Tang, H. 1995. "Evaluation of Smash Technique Using by Conservation of Angular Momentum." Bulletin of Toyama Prefectural University 5: 50-5.

[7] Tang, H. 1996. "Evaluation of Smash Technique from the Viewpoint of Conservation of Angular Momentum." Biomechanisms 13: 33-40.

[8] Jørgensen, U., and Winge, S. 1987. "Epidemiology of Badminton Injuries." International Journal of Sports
Medicine 8 (6): 379-82.

[9] Yung, P. S., Chan, R. H., Wong F. C., Cheuk, P. W., and Fong, D. T. 2007. "Epidemiology of Injuries in Hong Kong Elite Badminton Athletes." Research in Sports Medicine 15 (2): 133-46.

[10] Fahlström, M., Björnstig, U., and Lorentzon, R. 1998. "Acute Badminton Injuries." Scandinavian Journal of Medicine \& Science in Sports 8 (3): 145-8.

[11] Hensley, L. D., and Paup, D. C. 1979. "A Survey of Badminton Injuries." British Journal of Sports Medicine 13 (4): 156-60.

[12] Høy, K., Lindblad, B. E., Terkelsen, C. J., and Helleland, H. E. 1994. "Badminton Injuries-A Prospective Epidemiological and Socioeconomic Study." British Journal of Sports Medicine 28 (4): 276-9.

[13] Ogiuchi, T., Muneta, T., Yagishita, K., and Yamamoto, H. 1998. "Sports Injuries in Elite Badminton Players." Japanese Journal of Orthopaedic Sports Medicine 18 (4): 37-42.

[14] Jørgensen, U., and Winge, S. 1990. "Injuries in Badminton.” Journal of Sports Medicine 10 (1): 59-64.

[15] Kondrič, M., Matković, B., Furjan-Mandić, G., Hadžić, V., and Dervišević, E. 2011. "Injuries in Racket Sports among Slovenian Players." Journal of the Croatian Anthropological Society 35 (2): 413-7.

[16] Fahlström, M., Yeap, J. S., Alfredson, H., and Söderman, K. 2006. "Shoulder Pain-A Common Problem in World-Class Badminton Players." Scandinavian Journal of Medicine \& Science in Sports 16 (3): 168-73.

[17] Warashina, Y., Sasai, H., Nakata, Y., and Shiraki, H. 2015. "Descriptive Epidemiology of Shoulder Pain in Japanese Badminton Players." Research in Exercise Epidemiology 17 (1): 29-36.

[18] Ishii, T., Mukai, N., and Miyakawa, S. 2010. "Development of the Prediction System for the Onset of Throwing Shoulder Injury with Logistic Regression Analysis." Japanese Journal of Physical Fitness and Sports Medicine 59 (4): 389-94.

[19] Suzuki, H., Sawada, Y., and Sai, K. 1989. "Prevention of Sports Injuries during Badminton.” Journal of Clinical Sports Medicine 6 (extra): 421-42. 